\section{RSP}

http://www.rsp.fsp.usp.br/
Revista de Saúde Pública

\title{
Consumo alimentar segundo os dias da semana - Inquérito Nacional de Alimentação, 2008-2009
}

Luana Silva Monteiro', Bruna Kulik Hassan", Camilla Chermont Prochnik Estima", Amanda de Moura Souza"I, Eliseu Verly Junior ${ }^{\mathrm{IV}}$, Rosely Sichieri"', Rosangela Alves Pereirav

' Curso de Nutrição. Universidade Federal do Rio de Janeiro. Macaé, RJ, Brasil

" Programa de Pós-Graduação em Saúde Coletiva. Instituto de Medicina Social. Universidade do Estado do Rio de Janeiro. Rio de Janeiro, RJ, Brasil

III Instituto de Estudos em Saúde Coletiva. Universidade Federal do Rio de Janeiro. Rio de Janeiro, RJ, Brasil

iv Departamento de Epidemiologia. Instituto de Medicina Social. Universidade do Estado do Rio de Janeiro. Rio de Janeiro, RJ, Brasil

$\checkmark$ Departamento de Nutrição Social Aplicada. Instituto de Nutrição Josué de Castro. Universidade Federal do Rio de Janeiro. Rio de Janeiro, RJ, Brasil
Correspondência:

Luana Silva Monteiro Campus UFRJ-Macaé

Prof. Aloísio Teixeira

Rua Aloísio da Silva Gomes, 50 Granja dos Cavaleiros 27930-560 Macaé, RJ, Brasil E-mail: luananutrir@gmail.com

Recebido: 8 dez 2014 Aprovado: 2 out 2016

Como citar: Monteiro LS, Hassan BK, Estima CCP, Souza AM, Verly Jr E, Sichieri R, et al. Consumo alimentar segundo os dias da semana - Inquérito Nacional de Alimentação, 2008-2009. Rev Saude Publica. 2017;51:93.

Copyright: Este é um artigo de acesso aberto distribuído sob os termos da Licença de Atribuição Creative Commons, que permite uso irrestrito, distribuição e reprodução em qualquer meio, desde que o autor e a fonte originais sejam creditados.

\section{RESUMO}

OBJETIVO: Avaliar as variações na ingestão de energia, nutrientes e grupos de alimentos entre dias da semana e dias de fim de semana na população brasileira.

MÉTODOS: Foram utilizados dados obtidos no primeiro Inquérito Nacional de Alimentação (2008-2009) de um dia de registro alimentar de amostra representativa da população brasileira com 10 ou mais anos de idade $(n=34.003)$. Para as análises, foram considerados os pesos amostrais e o efeito do desenho de estudo. Foram estimadas as médias (e desvios-padrões) e frequências (\%) da ingestão de energia, nutrientes e grupos de alimentos para dias de semana (de segunda a sexta-feira) e de final de semana (sábado e domingo), sendo estimadas as diferenças segundo os dias da semana para os estratos populacionais analisados.

RESULTADOS: A ingestão diária média de energia do fim de semana foi $8 \%$ maior que a observada para dias de semana. A contribuição percentual média de carboidrato para a ingestão diária de energia foi maior durante a semana em comparação com sábado e domingo (56,3\% versus $54,1 \%$; $\mathrm{p}<0,01)$. O inverso foi observado para as médias da contribuição para a ingestão diária de energia de gordura total $(26,8 \%$ versus $28,4 \%)$, gordura saturada $(9,1 \%$ versus $9,9 \%)$ e gordura trans $(1,4 \%$ versus $1,6 \%)$. As mudanças mais importantes entre os dias de semana e de fim de semana foram observadas para ovos, bebidas com adição de açúcar, salgadinhos e chips, feijão e massas. Nos finais de semana, a frequência de consumo de bebidas com adição de açúcar aumentou em $34 \%$, a quantidade consumida sofreu incremento de $42 \%$ e a contribuição para a ingestão de energia se elevou em $62 \%$, quando comparados aos dias de semana.

CONCLUSÕES: A população brasileira aumenta a ingestão de energia e de marcadores de alimentação pouco saudável nos finais de semana em comparação aos dias de semana.

DESCRITORES: Consumo de Alimentos. Ingestão de Energia. Comportamento Alimentar. Inquéritos sobre Dietas. 


\section{INTRODUÇÃO}

Nas últimas décadas, mudanças expressivas ocorreram no consumo alimentar em diversos países ${ }^{1}$. No Brasil, tem sido observado aumento da participação de produtos processados na disponibilidade de alimentos nos domicílios brasileiros ${ }^{18}$. Dados das Pesquisas de Orçamentos Familiares mostraram que, entre 2002-2003 e 2008-2009, a participação relativa de biscoitos, refrigerantes e refeições prontas na disponibilidade de energia no domicílio aumentou em $10 \%, 16 \%$ e $40 \%$, respectivamente ${ }^{16}$. A alimentação no Brasil vem se configurando como sendo de alta densidade energética, elevado conteúdo de açúcares e sódio e baixo teor de fibras e micronutrientes. Dessa forma, nossa dieta constitui importante fator de risco para o aumento da prevalência de obesidade e de doenças crônicas não transmissíveis ${ }^{20,25}$.

Destaca-se que os dias da semana são classicamente considerados como fontes de variabilidade intraindividual do consumo alimentar ${ }^{2}$, considerada importante na avaliação do erro nas estimativas do consumo de alimentos e de nutrientes em inquéritos alimentares baseados em inquérito recordatório de 24 horas e em diário alimentar ${ }^{29}$.

Estudos que avaliaram o consumo alimentar segundo os dias da semana relatam que o consumo alimentar nos finais de semana apresenta pior qualidade nutricional do que nos dias de semana, por maior consumo de refrigerantes e outras bebidas açucaradas, bebidas alcoólicas e gorduras, e menor participação dos alimentos integrais, levando à ingestão mais elevada de energia nos finais de semana ${ }^{10,23,24}$.

Ademais, o incremento sistemático na ingestão energética pode levar ao balanço energético positivo e favorecer o ganho de peso, como mostrado por Brown et al. ${ }^{4}$ Esses autores, ao avaliarem mulheres de meia idade incluídas no Australian Longitudinal Study on Women's Health, observaram que desequilíbrio no balanço energético de apenas $10 \mathrm{kcal}$ por dia se associou ao ganho de peso médio de aproximadamente $0,5 \mathrm{~kg}$ por ano ${ }^{4}$.

São escassos estudos que avaliam variações nos hábitos de consumo alimentar segundo os dias da semana, particularmente no Brasil. A caracterização do consumo alimentar segundo os da semana pode contribuir para o refinamento da avaliação do consumo de alimentos e fundamentar ações de promoção da saúde e da alimentação saudável. O presente estudo teve como objetivo estimar as variações na ingestão de energia, nutrientes e grupos de alimentos entre dias da semana e dias de fim de semana na população brasileira.

\section{MÉTODOS}

Foram utilizados os dados do primeiro Inquérito Nacional de Alimentação (INA) incluído como um módulo da Pesquisa de Orçamentos Familiares (POF 2008-2009), desenvolvida pelo Instituto Brasileiro de Geografia e Estatística (IBGE). O INA foi realizado em subamostra dos domicílios incluídos na POF 2008-2009 e um total 34.003 moradores com pelo menos 10 anos de idade participaram do inquérito ${ }^{11}$.

O consumo alimentar foi estimado por meio de dois registros alimentares não consecutivos obtidos no período de uma semana. Os participantes foram orientados a informar todos os alimentos e bebidas (exceto água) consumidos, a quantidade consumida, o dia da semana, e o horário e o local de consumo (dentro ou fora do domicílio) ${ }^{11}$. Neste estudo, foram utilizados os dados do primeiro dia de registro alimentar da população avaliada.

A ingestão de energia, carboidrato, lipídio e proteína, gordura saturada, gordura trans e açúcar de adição foi estimada com base na tabela de composição dos alimentos consumidos no Brasil ${ }^{13}$.

Os alimentos citados nos registros alimentares foram categorizados em 58 grupos, de acordo com agrupamento adotado por Pereira et al. ${ }^{21}$ Resumidamente, esse sistema de agrupamento considera nove grupos principais desagregados em subgrupos de acordo com 
as características nutricionais de alimentos e bebidas e a sua utilização na dieta. Muitos subgrupos considerados nessa categorização foram referidos em proporções muito baixas no INA $(<2 \%)$. Para a análise da variação na prevalência de consumo entre dias de semana e de final de semana, optou-se por avaliar somente os subgrupos de alimentos referidos por pelo menos 10\% da população (20 subgrupos) (Quadro).

Os relatos entre segunda e sexta-feira foram considerados como dias de semana, e os de sábado e domingo foram tratados como consumo de final de semana (FDS). A inclusão da sexta-feira nos dias de semana foi baseada em análises exploratórias que permitiram identificar que, na população estudada, o consumo de energia e macronutrientes referente à sexta-feira foi mais compatível com o consumo de segunda a quinta-feira do que o de sábado e domingo. Os dados foram avaliados por sexo, faixa etária e por renda familiar per capita. Na categorização por faixa etária, consideramos adolescentes os indivíduos com idades entre 10 e 19 anos, adultos os que tinham entre 20 e 59 anos e idosos aqueles com 60 anos ou mais.

Para estimar a renda familiar per capita, o rendimento total do domicílio foi dividido pelo número de moradores. A renda familiar foi categorizada em cinco faixas: $<0,5$ salário mínimo per capita, 0,5 $\leq$ salário mínimo per capita $<1,1 \leq$ salários mínimos per capita $<2,2$

Quadro. Grupos de alimentos citados pela população brasileira. Inquérito Nacional de Alimentação (INA).

\begin{tabular}{|c|c|}
\hline Grupos de alimentos & Alimentos citados \\
\hline Ovos & Ovo de galinha, ovo de codorna e omelete \\
\hline Bebidas adoçadas com açúcar & Refrigerantes, xarope de guaraná, refresco e sucos em pó \\
\hline Salgadinhos e chips & Rosquinha salgada, biscoito salgado, chips (salgadinhos), pipoca doce ou salgada \\
\hline Feijão & Feijões, lentilha, grão de bico, soja \\
\hline Massas & Macarrões, lasanha, capeletti, canelone, nhoque, ravióli, yakissoba \\
\hline Suco de fruta & $\begin{array}{l}\text { Suco de abacaxi, suco de acerola, suco de cupuaçu, suco de goiaba, suco de laranja, suco de laranja com banana, } \\
\text { suco de mamão, suco de manga, suco de maracujá, suco de morango, suco de pêssego, caldo de cana, suco orgânico }\end{array}$ \\
\hline Óleos e gorduras & Manteiga ou margarina com sal ou sem sal, manteiga de garrafa, toucinho, pele de porco preparada (pururuca), azeite \\
\hline Hortaliças & $\begin{array}{l}\text { Abóbora, abobrinha, acelga, agrião, alface, alho, alho-poró, almeirão, aspargo, berinjela, bertalha, beterraba, brócolis, broto } \\
\text { de alfafa, broto de feijão, cebola, cebolinha, cenoura, chicória, cogumelo, chuchu, couve, couve-flor, ervilha em vagem, } \\
\text { escarola, espinafre, palmito, jiló, pepino, pimentão, quiabo, rabanete, repolho, rúcula, taioba, tomate, vagem, vinagreira }\end{array}$ \\
\hline Frutas & $\begin{array}{l}\text { Abacate, Abacaxi, abiu, açaí, acerola, ameixa, amora, atemoia, bacuri, bananas, biribá, cajá-manga, caju, cana de } \\
\text { açúcar, caqui, carambola, cereja, seriguela, cupuaçu, figo, fruta do conde, goiaba, graviola, imbu, ingá, jabuticaba, } \\
\text { jaca, jambo, jamelão, jenipapo, jurubeba, kiwi, laranja, limão, maçã, mamão, manga, mangaba, maracujá, melancia, } \\
\text { melão, mexerica, murici, morango, passas, pequi, pêra, pêssego, pitanga, pitomba, romã, sapoti, tanja, umbu, uva }\end{array}$ \\
\hline Queijo & $\begin{array}{l}\text { Queijo prato, queijo colonial, queijo muçarela, queijo do reino, queijo minas, queijo coalho, queijo canastra, queijo } \\
\text { ricota, queijo polenguinho, queijo ralado, queijo gorgonzola, requeijão, queijo não especificado }\end{array}$ \\
\hline Carnes processadas & $\begin{array}{l}\text { Apresuntado, blaquet de peru, carne de sol, carne seca, chouriço, linguiça, mortadela, pasta de carne em conserva, } \\
\text { patê, peito de peru, presunto, salame, salsicha, tender }\end{array}$ \\
\hline $\begin{array}{l}\text { Aves e preparações à base de } \\
\text { aves }\end{array}$ & Galinha ou frango, chester, codorna, moela de frango, fígado de frango, hambúrguer de frango, pato, peru \\
\hline Pães & $\begin{array}{c}\text { Pão com manteiga/margarina, pão de hambúrguer, pão de forma, pão de sal, bisnaguinha, pão integral, pão não } \\
\text { especificado, torrada de qualquer pão }\end{array}$ \\
\hline Arroz & $\begin{array}{c}\text { Arroz (polido, parboilização, agulha, agulhinha, etc.), amido de arroz, arroz à grega, arroz carreteiro, arroz com feijão, } \\
\text { arroz com mandioca, arroz com ovo, arroz com leite, arroz integral, aveia em flocos, creme de arroz, mingau (arroz, } \\
\text { fubá, aveia, farinha, etc.), creme de arroz, risoto }\end{array}$ \\
\hline $\begin{array}{l}\text { Carnes e preparações à base } \\
\text { de carnes }\end{array}$ & Carne bovina, caprina, paca, bode, cabrito, capivara, carneiro, jacaré, suína \\
\hline Doces e sobremesas & $\begin{array}{l}\text { Alfajor, biscoito, biscoito doce, biscoito recheado, bolos, brevidade, panetone, churros, cuca, cavaca, filhós, pão de } \\
\text { mel, pão doce, pavê, rabanada, rocambole, rosca doce, quebra-queixo, rosca doce, sequilho, sonho, tortas }\end{array}$ \\
\hline Café e chá & Mate tradicional, erva-mate, chimarrão, chá, mate orgânico, chá com farinha, café solúvel, cappuccino, café, café com leite \\
\hline Balas e chocolates & $\begin{array}{l}\text { Amendoim achocolatado, bala, beijo de moça, barra de chocolate, bombom, brigadeiro, canudinho recheado, } \\
\text { chiclete, cocada, doce à base de ovos, doce de amendoim, doce de frutas cristalizadas, doces de frutas, geladinho, } \\
\text { goiabada, jujuba, maria mole, merengue, paçoca, pé de moleque, picolé, pirulito, torrone, suspiro }\end{array}$ \\
\hline Raízes e tubérculos & Aipim, batata, batata doce, cenoura, inhame, mandioquinha, milho, tapioca \\
\hline Leite & $\begin{array}{l}\text { Leite de vaca integral, leite de vaca fresco, leite de cabra, leite em pó integral, leite em pó desnatado, leite em pó, } \\
\text { leite fermentado, leite com sabor, leite com achocolatado, leite aromatizado, leite de vaca desnatado, leite de vaca } \\
\text { semidesnatado, leite não especificado pasteurizado }\end{array}$ \\
\hline
\end{tabular}


$\leq$ salários mínimos per capita < 5 e $\geq 5$ salários mínimos per capita. Para essa classificação, considerou-se o valor do salário mínimo de $\mathrm{R} \$ 415,00$ (quatrocentos e quinze reais) vigente em 15 de janeiro de 2009, data de referência da pesquisa ${ }^{12}$.

Os dados foram analisados com o uso do software Statistical Package for the Social Sciences (SPSS), versão 19. Nas análises, foram considerados os pesos amostrais e o efeito do desenho amostral.

As variações na ingestão de energia e nutrientes entre os estratos populacionais foram estimadas por meio de modelos lineares generalizados (GLM) desenvolvidos no módulo Complex Sample, com correção para Bonferroni na comparação segundo a classe de renda. Avaliamos as diferenças entre os estratos populacionais das variáveis categóricas por meio do teste qui-quadrado.

O protocolo da pesquisa foi aprovado pelo Comitê de Ética do Instituto de Medicina Social da Universidade do Estado do Rio de Janeiro (CAAE 0011.0.259.000-11, de 19 de julho de 2011).

\section{RESULTADOS}

Somente os indivíduos com idades $\geq 60$ anos não modificaram sua ingestão dietética entre os dias de semana e de FDS para nenhum dos componentes analisados. Já nos adultos, foram observadas mudanças para todos os fatores da dieta avaliados. Entre os adolescentes, foram observadas redução na contribuição dos carboidratos para a ingestão de energia e aumento na participação de lipídios totais e gordura trans na ingestão diária de energia. Para as mulheres, apenas para o açúcar de adição não foram observadas variações. Para os homens, além do açúcar de adição, também não houve variação para os carboidratos.

Observou-se aumento médio de 8\% na ingestão diária de energia no FDS quando comparado aos dias de semana (2.059 kcal versus $1.906 \mathrm{kcal} ; \mathrm{p}<0,01$ ). Diferenças significativas na ingestão diária de energia entre dias de semana e de FDS foram observadas para mulheres e homens, adultos e indivíduos com renda per capita acima de dois salários mínimos mensais. Essa diferença foi mais expressiva entre os indivíduos que pertenciam às famílias com renda mensal $\geq 5$ salários mínimos per capita, para os quais foi observado incremento médio da ordem de $16 \%$ na ingestão energética diária, o que foi 2,3 vezes maior do que o aumento médio observado na população em geral (Tabela 1).

A contribuição percentual dos carboidratos para a ingestão diária de energia foi menor no FDS, e essa redução foi, em média, de 4\%, não sendo observadas oscilações importantes entre os diferentes estratos. Contudo, para os homens, os idosos, e indivíduos com renda mensal $\geq 5$ salários mínimos per capita, não houve variação na proporção da energia diária fornecida pelos carboidratos (Tabela 1).

A contribuição percentual dos lipídios totais, gordura saturada e gordura trans no FDS foi mais elevada do que nos dias de semana. Essas mudanças ocorreram de forma similar em todos os estratos analisados (sexo, faixa de idade e renda). Os incrementos mais expressivos na contribuição dos lipídios totais, gordura saturada e gordura trans para a ingestão diária de energia foram observadas para a faixa de renda familiar mensal abaixo de 0,5 salário mínimo per capita. Nesse grupo, a contribuição dos lipídios totais para a energia diária aumentou, em média, $10 \%$ entre os dias de semana e de FDS, passando de $25 \%$ para $28 \%(\mathrm{p}<0,01)$; a gordura saturada, durante os dias da semana correspondia, em média a 7,9\% da ingestão diária de energia e, durante os dias de FDS, a 9,1\%, o que representou um aumento médio de $13 \%$ ( $p<0,01$ ); para a gordura trans, o incremento médio segundo os dias da semana foi da ordem de $29 \%$ ( $p<0,01)$ e essa taxa foi maior que o dobro do aumento médio observado na população em geral (Tabela 1).

Observou-se que a contribuição do açúcar de adição para a ingestão diária de energia aumentou no FDS em relação aos dias de semana e os incrementos mais significativos ocorreram entre os indivíduos de famílias com renda mensal per capita $\geq 5$ salários mínimos. Nesse grupo, 
Tabela 1. Ingestão de energia e contribuição (\%) de macronutrientes para a ingestão diária de energia nos dias de semana e final de semana de acordo com as características socioeconômicas. Inquérito Nacional de Alimentação, Brasil, 2008-2009.

\begin{tabular}{|c|c|c|c|c|c|c|c|c|c|c|c|c|}
\hline \multirow{3}{*}{ Grupo } & \multirow{2}{*}{\multicolumn{2}{|c|}{$\frac{\text { Energia }}{\text { (kcal) }}$}} & \multirow{2}{*}{\multicolumn{2}{|c|}{$\frac{\text { Carboidratos }}{(\%)}$}} & \multirow{2}{*}{\multicolumn{2}{|c|}{$\frac{\text { Lipídios totais }}{(\%)}$}} & \multirow{2}{*}{\multicolumn{2}{|c|}{$\frac{\text { Gordura saturada }}{(\%)}$}} & \multirow{2}{*}{\multicolumn{2}{|c|}{$\frac{\text { Gordura trans }}{(\%)}$}} & \multirow{2}{*}{\multicolumn{2}{|c|}{$\frac{\text { Açúcar de adição }}{(\%)}$}} \\
\hline & & & & & & & & & & & & \\
\hline & SE & FDS & SE & FDS & SE & FDS & SE & FDS & SE & FDS & SE & FDS \\
\hline Brasil (total) & $1.906^{\mathrm{a}}$ & 2.059 & $56^{a}$ & 54 & $27^{\mathrm{a}}$ & 28 & $9,2^{\mathrm{a}}$ & 10,0 & $1,4^{\mathrm{a}}$ & 1,6 & $13^{\mathrm{a}}$ & 14 \\
\hline Variação FDS-SE (\%) & \multicolumn{2}{|c|}{7} & \multicolumn{2}{|c|}{-4} & \multicolumn{2}{|c|}{6} & \multicolumn{2}{|c|}{8} & \multicolumn{2}{|c|}{13} & \multicolumn{2}{|c|}{4} \\
\hline \multicolumn{13}{|l|}{ Sexo } \\
\hline Feminino & $1.710^{\mathrm{b}}$ & 1.879 & $57^{\mathrm{b}}$ & 55 & $27^{\mathrm{a}}$ & 29 & $9,3^{b}$ & 10,1 & $1,4^{\mathrm{b}}$ & 1,6 & 14 & 15 \\
\hline Variação FDS-SE (\%) & \multicolumn{2}{|c|}{9} & \multicolumn{2}{|c|}{-4} & \multicolumn{2}{|c|}{7} & \multicolumn{2}{|c|}{8} & \multicolumn{2}{|c|}{13} & \multicolumn{2}{|c|}{5} \\
\hline Masculino & $2.117^{\mathrm{b}}$ & 2.250 & 56 & 54 & $27^{b}$ & 28 & $9,0^{\mathrm{b}}$ & 9,8 & $1,3^{\mathrm{a}}$ & 1,5 & 13 & 13 \\
\hline Variação FDS-SE (\%) & \multicolumn{2}{|c|}{6} & \multicolumn{2}{|c|}{-4} & \multicolumn{2}{|c|}{4} & \multicolumn{2}{|c|}{8} & \multicolumn{2}{|c|}{13} & \multicolumn{2}{|c|}{0} \\
\hline \multicolumn{13}{|l|}{ Faixa etária } \\
\hline Adolescente (10-19 anos) & 2.058 & 2.055 & $57^{\mathrm{a}}$ & 55 & $27^{b}$ & 29 & 9,3 & 10,3 & $1,5^{\mathrm{a}}$ & 1,7 & 15 & 15 \\
\hline Variação FDS-SE (\%) & \multicolumn{2}{|c|}{0} & \multicolumn{2}{|c|}{-4} & & & & & & & & \\
\hline Adulto (20-59 anos) & $1.913^{\mathrm{b}}$ & 2.107 & $56^{b}$ & 54 & $27^{b}$ & 28 & $9,1^{\mathrm{b}}$ & 9,9 & $1,3^{b}$ & 1,5 & $13^{\mathrm{a}}$ & 14 \\
\hline Variação FDS-SE (\%) & & & & & & & & & & & & \\
\hline Idoso ( $\geq 60$ anos) & 1.633 & 1.727 & 56 & 54 & 27 & 28 & 9,2 & 9,6 & 1,3 & 1,4 & 11 & 11 \\
\hline Variação FDS-SE (\%) & & & & & & & & & & & & \\
\hline Renda familiar per capita (salári & & & & & & & & & & & & \\
\hline$<0,5$ & 1.791 & 1.800 & $58^{\mathrm{a}}$ & 56 & $25^{b}$ & 28 & $7,9^{b}$ & 9,1 & $1,2^{b}$ & 1,7 & 12 & 13 \\
\hline Variação FDS-SE (\%) & & & & & & & & & & & & \\
\hline $0,5-1$ & 1.901 & 2.069 & $57^{\mathrm{a}}$ & 54 & 26 & 28 & 8,8 & 9,6 & $1,4^{\mathrm{a}}$ & 1,6 & 13 & 13 \\
\hline Variação SE versus FDS (\%) & & & & & & & & & & & & \\
\hline $1-2$ & 1.927 & 2.011 & $56^{b}$ & 54 & 27 & 28 & 9,3 & 9,6 & 1,4 & 1,5 & 13 & 13 \\
\hline Variação SE versus FDS (\%) & & & & & & & & & & & & \\
\hline $2-5$ & $1.975^{\mathrm{b}}$ & 2.018 & $55^{a}$ & 54 & $28^{\mathrm{b}}$ & 30 & $9,9^{\mathrm{a}}$ & 10,7 & 1,5 & 1,4 & $14^{\mathrm{a}}$ & 15 \\
\hline Variação FDS-SE (\%) & & & & & & & & & & & & \\
\hline$\geq 5$ & $1.917^{\mathrm{b}}$ & 2.277 & 54 & 53 & 28 & 29 & 10,6 & 11,4 & 1,2 & 1,3 & $13^{\mathrm{a}}$ & 15 \\
\hline Variação FDS-SE (\%) & & & & & & & & & & & & \\
\hline
\end{tabular}

SE: dias de semana (de segunda a sexta-feira); FDS: finais de semana (sábado e domingo)

$\%$ Variação: (FDS-SE)/SE*100

a $\mathrm{p}<0,05$

${ }^{\mathrm{b}} \mathrm{p}<0,01$

a participação do açúcar de adição na ingestão diária de energia entre os dias de semana e de FDS aumentou de $13 \%$, em média, para um valor médio de $15 \%$, o que representou incremento médio de $16 \%(\mathrm{p}<0,05)$. Esse incremento foi quatro vezes mais elevado do que o aumento médio na população em geral (Tabela 1).

Os alimentos mais consumidos e os que representaram maior contribuição calórica para a ingestão diária de energia tanto nos dias de semana quanto no FDS foram o arroz, café ou chá, feijão, pão, e carnes. Os grupos de alimentos cuja prevalência de consumo variaram de forma mais importante ( $>20 \%$ de variação) entre os dias de semana e de FDS foram: ovos (-35\%), bebidas com adição de açúcar (+34\%), salgadinhos e chips (-22\%), feijão (-21\%) e massas $(+20 \%)$ (Tabela 2).

Para os homens, entre os dias de semana e de FDS, observou-se redução da prevalência de consumo de arroz e feijão e elevação na prevalência de consumo óleos e gorduras, ovos e cerveja. Para as mulheres, houve redução da prevalência de consumo de feijão, hortaliças, sucos de frutas, e salgadinhos e chips. Tanto para os homens como para as mulheres, a prevalência de consumo das bebidas com adição de açúcar e de massas aumentou no FDS em comparação aos dias de semana. A prevalência de consumo de feijão foi reduzida para todos os grupos etários. Entre os adultos, entre os dias de semana e de FDS, a prevalência de 
consumo dos óleos e gorduras, massas, e cerveja aumentou; já a prevalência do consumo de arroz, hortaliças, ovos, e milho e preparações com milho diminuiu. Entre os dias da semana e de FDS, adolescentes e adultos também reduziram a prevalência de consumo de sucos de frutas e salgadinhos e chips e idosos apenas aumentaram a frequência de consumo dos óleos e gorduras. Verificou-se que a cerveja figurava entre os 20 alimentos mais consumidos por adultos e homens durante o FDS e que seu consumo no FDS era três vezes maior do que o estimado para os dias de semana (dados não mostrados).

Comparando o consumo alimentar entre os dias de semana e deFDS foram verificadas mudanças na contribuição calórica dos alimentos para a ingestão diária de energia. As modificações mais relevantes (> 20\%) foram observadas para as bebidas com adição de açúcar (+62\%), ovos (-33\%), massas (+30\%), feijão (-23\%), sucos de frutas (-21\%) e salgadinhos e chips (-21\%), além desses também houve redução da contribuição calórica de frutas, hortaliças e arroz (Tabela 2). Para ovos, feijão, sucos de frutas, hortaliças, frutas e arroz houve redução tanto na prevalência de consumo como nas quantidades consumidas como na contribuição calórica entre os dias de semana e de FDS, sendo as reduções mais importantes observadas para os ovos e o feijão. Por outro lado, entre dias de semana e de FDS, para as bebidas com adição de açúcar, massas e óleos e gorduras houve incremento tanto na prevalência de consumo quanto nas quantidades consumidas e na contribuição para a ingestão energética. Deve ser salientado que a importante variação observada para as bebidas com adição de açúcar, com aumento $42 \%$ nas quantidades consumidas e de $62 \%$ na contribuição para a ingestão diária de energia (Tabela 2).

Tabela 2. Comparação entre o consumo de grupos alimentares entre os dias de semana (SE) e final de semana (FDS). Inquérito Nacional de Alimentação, Brasil, 2008-2009.

\begin{tabular}{|c|c|c|c|c|c|c|c|c|c|}
\hline \multirow{3}{*}{ Grupo } & \multicolumn{3}{|c|}{ Prevalência de consumo } & \multicolumn{3}{|c|}{ Quantidade consumida } & \multicolumn{3}{|c|}{$\begin{array}{c}\text { Contribuição para a ingestão } \\
\text { diária de energia }{ }^{a}\end{array}$} \\
\hline & SE & FDS & $\begin{array}{l}\text { Variação } \\
\text { FDS-SE }\end{array}$ & SE & FDS & $\begin{array}{l}\text { Variação } \\
\text { FDS-SE }\end{array}$ & SE & FDS & $\begin{array}{l}\text { Variação } \\
\text { FDS-SE }\end{array}$ \\
\hline & $\%$ & $\%$ & $\%$ & gramas & gramas & $\%$ & $\%$ & $\%$ & $\%$ \\
\hline Ovos & $17^{\mathrm{b}}$ & $12^{\mathrm{b}}$ & -35 & $9,2^{\mathrm{b}}$ & $6,6^{\mathrm{b}}$ & -39 & $1,5^{c}$ & $1,0^{c}$ & -33 \\
\hline Bebidas com adição de açúcar & $29^{b}$ & $44^{\mathrm{b}}$ & 34 & $101,7^{\mathrm{b}}$ & $174,5^{\mathrm{b}}$ & 42 & $2,1^{\mathrm{c}}$ & $3,4^{c}$ & 62 \\
\hline Salgadinhos e chips & $19^{c}$ & $16^{c}$ & -22 & $4,3^{b}$ & $4,6^{b}$ & 7 & $1,9^{b}$ & $1,5^{b}$ & -21 \\
\hline Feijão & $78^{\mathrm{b}}$ & $65^{\mathrm{b}}$ & -21 & $134,7^{\mathrm{b}}$ & $103,6^{\mathrm{b}}$ & -30 & $10,5^{c}$ & $8,1^{\mathrm{c}}$ & -23 \\
\hline Massas & $21^{c}$ & $26^{c}$ & 20 & $28,9^{c}$ & $41,2^{\mathrm{c}}$ & 30 & $3,3^{\mathrm{b}}$ & $4,3^{b}$ & 30 \\
\hline Suco de frutas & $34^{\mathrm{c}}$ & $29^{c}$ & -18 & $109,6^{c}$ & $92,2^{\mathrm{c}}$ & -19 & $4,8^{c}$ & $3,8^{\mathrm{c}}$ & -21 \\
\hline Óleos e gorduras & $37^{\mathrm{b}}$ & $43^{b}$ & 14 & $2,5^{c}$ & $2,9^{c}$ & 14 & $2,3^{\mathrm{b}}$ & $2,7^{b}$ & 17 \\
\hline Hortaliças & $43^{c}$ & $37^{c}$ & -14 & $27,6^{\mathrm{b}}$ & $24,9^{\mathrm{b}}$ & -11 & $0,6^{\mathrm{b}}$ & $0,5^{\mathrm{b}}$ & -11 \\
\hline Frutas & $35^{\mathrm{b}}$ & $31^{\mathrm{b}}$ & -13 & $66,3^{\mathrm{b}}$ & $59,0^{\mathrm{b}}$ & -12 & $3,1^{\mathrm{b}}$ & $2,6^{\mathrm{b}}$ & -16 \\
\hline Queijos e produtos à base de queijo & 14 & 15 & 11 & 4,1 & 4,8 & 15 & 1,0 & 1,1 & 10 \\
\hline $\begin{array}{l}\text { Carnes processadas e preparações à base de } \\
\text { carnes processadas }\end{array}$ & 19 & 21 & 9 & 6,3 & 5,9 & -7 & 1,9 & 1,7 & -11 \\
\hline Aves e preparações à base de aves & 27 & 29 & 7 & 22,8 & 26,8 & 15 & 4,8 & 4,7 & -2 \\
\hline Pão & 64 & 67 & 5 & 38,6 & 40,4 & 4 & 8,8 & 8,9 & 1 \\
\hline Arroz & $91^{\mathrm{c}}$ & $88^{c}$ & -4 & $91,4^{\mathrm{b}}$ & $80,4^{\mathrm{b}}$ & -14 & $14,8^{\mathrm{c}}$ & $13,1^{\mathrm{c}}$ & -11 \\
\hline Carnes e preparações à base de carnes & 53 & 55 & 4 & 47,5 & 50,8 & 6 & 10,0 & 10,7 & 7 \\
\hline Doces e sobremesas & 28 & 27 & 3 & $16,7^{\mathrm{b}}$ & $20,6^{\mathrm{b}}$ & 19 & 4,9 & 5,2 & 6 \\
\hline Café e chá & 82 & 81 & 1 & 233,7 & 227,7 & -3 & 6,5 & 6,3 & -3 \\
\hline Balas e chocolates & 12 & 12 & 0 & 6,2 & 6,7 & 7 & 1,1 & 1,2 & 12 \\
\hline Raízes e tubérculos & 27 & 27 & 0 & 19,3 & 18,1 & -7 & 3,8 & 4,0 & 5 \\
\hline Leite & 23 & 23 & 0 & 57,9 & 63,6 & 9 & 2,4 & 2,5 & 4 \\
\hline
\end{tabular}

SE: dias de semana (de segunda a sexta-feira); FDS: finais de semana (sábado e domingo)

${ }^{a}$ Contribuição para a ingestão diária de energia - (energia do grupo de alimento*100) / ingestão energética total.

${ }^{\mathrm{b}} \mathrm{p}<0,05$

${ }^{c} \mathrm{p}<0,01$ 
Entre os dias de semana e de FDS, houve redução nas quantidades médias consumidas de ovos, feijão, sucos de frutas, arroz, frutas e hortaliças; em contrapartida, observou-se incremento nas quantidades médias consumidas de bebidas com adição de açúcar, massas, doces e sobremesas à base de farinhas, óleos e gorduras e salgadinhos e chips. Deve ser destacado que as variações mais significativas ( $>20 \%$ de variação) nas quantidades consumidas foram observadas para as bebidas com adição de açúcar (+42\%), ovos (-39\%), feijão (-30\%) e massas $(+30 \%)$ (Tabela 2).

Nas Figuras 1 e 2, estão representadas as variações entre os dias de semana e de FDS registradas para as bebidas com adição de açúcar e o feijão, visto que esses grupos apresentaram as variações mais expressivas e sofreram modificações significativas para quase todas as categorias das variáveis explanatórias analisadas. Em comparação com os dias de semana, o aumento estatisticamente significativo na frequência de consumo de bebidas com adição de açúcar no FDS foi observado em todos os estratos analisados, exceto para idosos. Para o feijão, a redução na frequência de consumo entre os dias de semana e de FDS também foi consistente para todos os estratos avaliados, exceto para os indivíduos que pertenciam às famílias com renda mensal < 0,5 salário mínimo per capita (Figura 1, A).

Observou-se variações entre os dias de semana e de FDS na contribuição calórica para a ingestão diária de energia das bebidas com adição de açúcar e do feijão. Tais como, redução na contribuição calórica média do feijão (10,5 versus $8,9 \%$; $p<0,01)$, exceto entre os indivíduos com renda mensal entre 1 e 2 e $\geq 5$ salários mínimos per capita (Figura 2, B). Para as bebidas com adição de açúcar, a contribuição calórica só não aumentou para os indivíduos com renda mensal < 0,5 salários mínimos per capita (Figura 2, A).

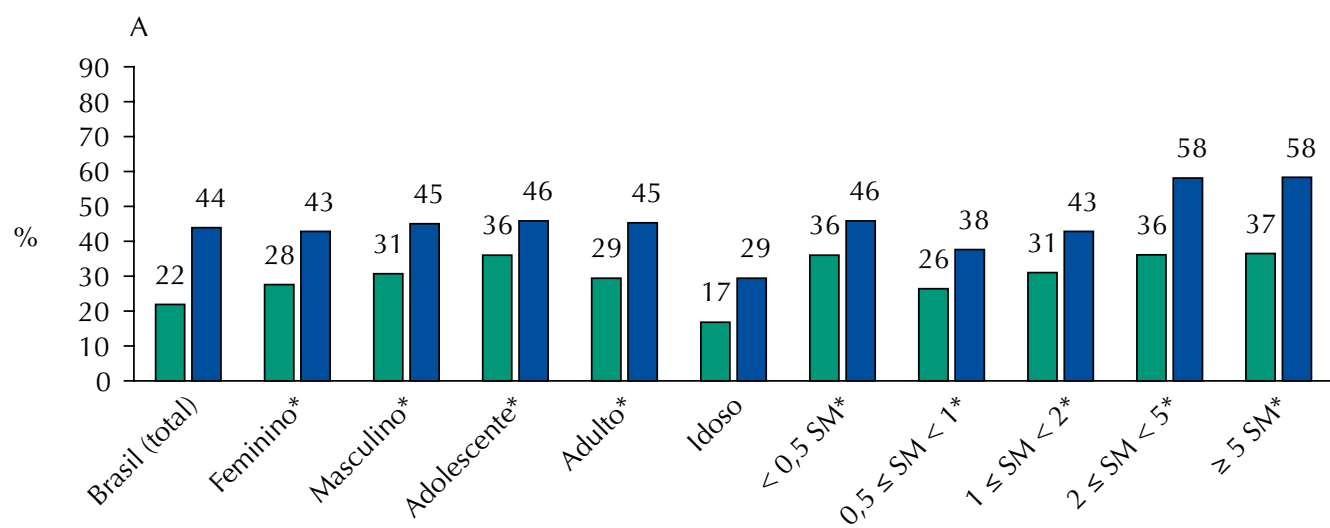

B

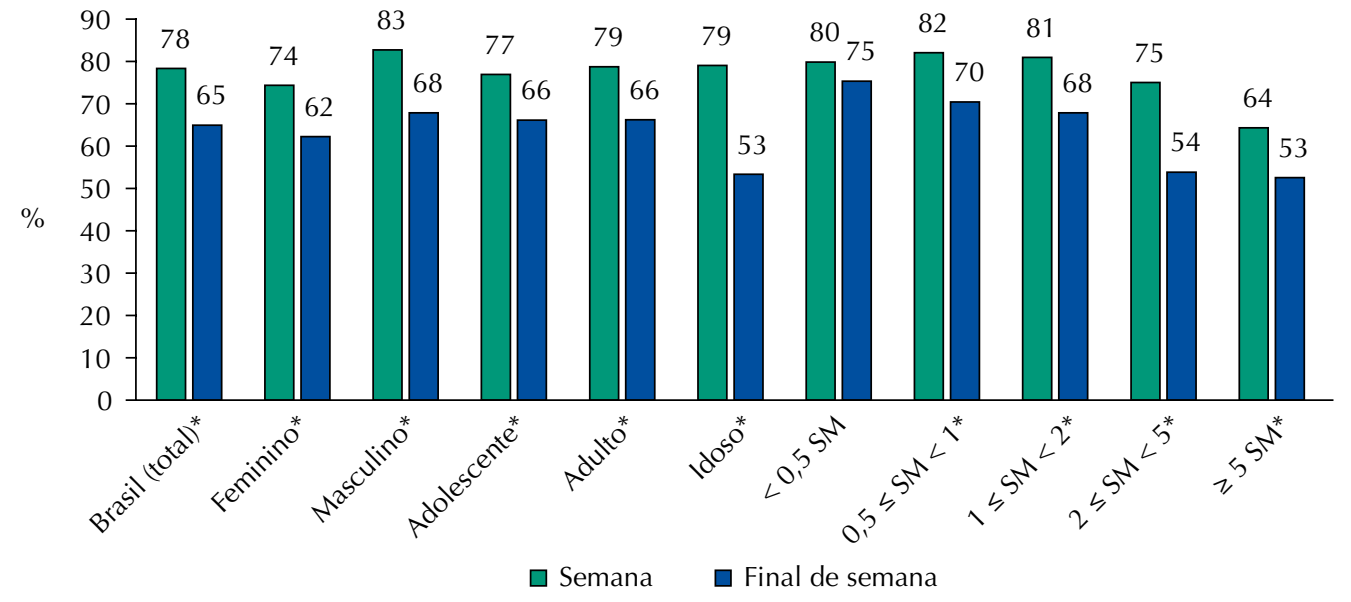

SM: salário mínimo

$* p<0,01$

Figura 1. Prevalência de consumo das bebidas com adição de açúcar (A) e feijão (B), em dias de semana e de final de semana. Inquérito Nacional de Alimentação, Brasil, 2008-2009. 

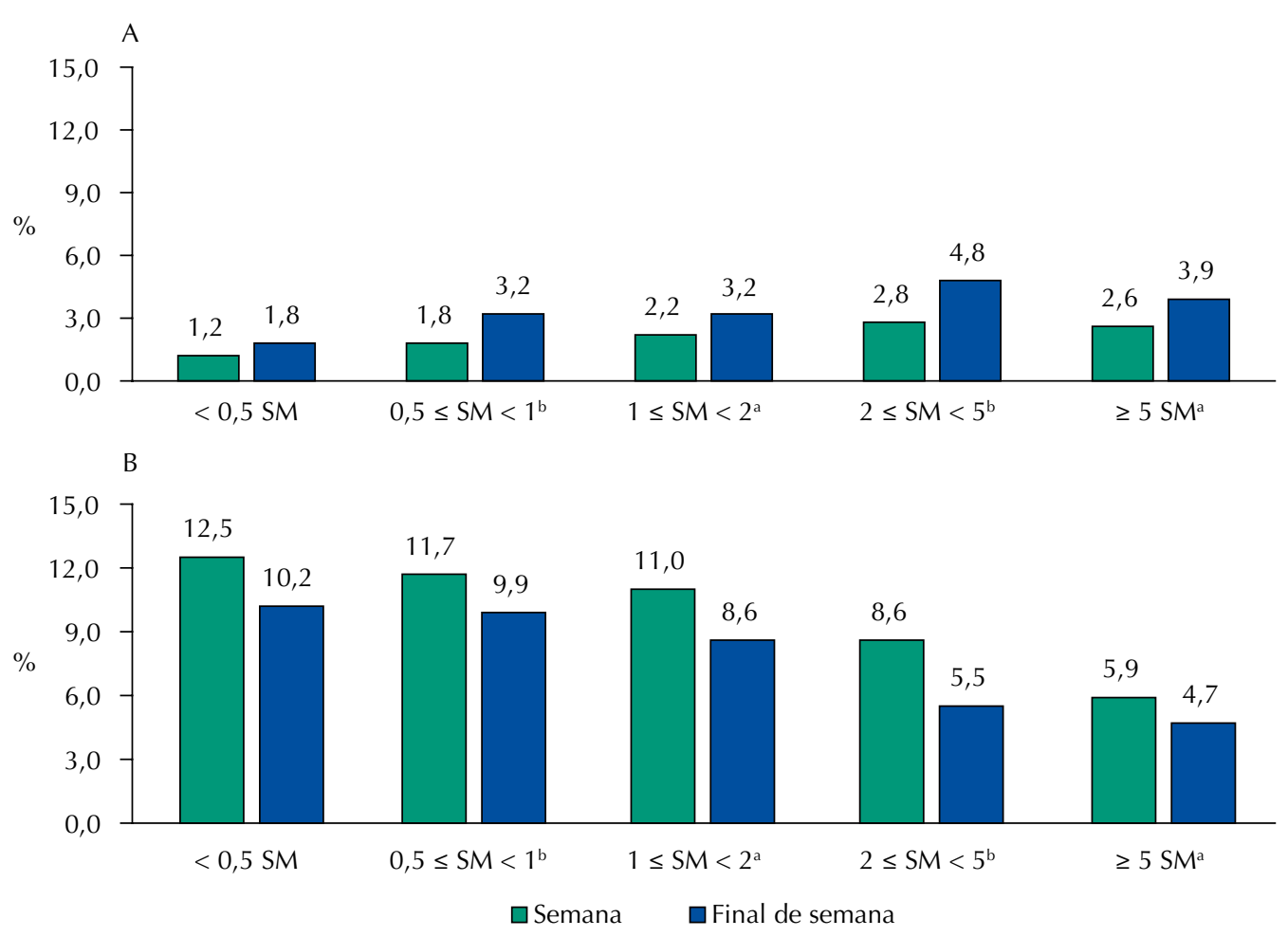

SM: salário mínimo

a $p<0,05$

${ }^{\mathrm{b}} \mathrm{p}<0,01$

Figura 2. Contribuição (\%) das bebidas com adição de açúcar (A) e feijão (B) para ingestão energética diária, em dias de semana e de final de semana de acordo com a renda. Inquérito Nacional de Alimentação, Brasil, 2008-2009.

\section{DISCUSSÃO}

Esse é o primeiro trabalho que compara a ingestão de energia, nutrientes e alimentos entre dias de semana e de FDS em amostra representativa da população brasileira. Este estudo mostrou que nos FDS ocorrem mudanças significativas no consumo alimentar em comparação com os dias de semana. No FDS, ocorreu consumo mais elevado de energia, lipídios, gorduras saturadas e trans e açúcar de adição. Esses achados podem estar relacionados à redução no FDS da frequência e das quantidades consumidas de grupos de alimentos que são parte do padrão alimentar tradicional da alimentação brasileira e de marcadores de alimentação saudável (nomeadamente, arroz, feijão, frutas e hortaliças), aliada ao aumento do consumo de alimentos como bebidas com adição de açúcar, óleos e gorduras, e massas.

Pesquisas conduzidas nos Estados Unidos e na Finlândia ${ }^{5,10,14}$ observaram resultados semelhantes aos do presente estudo, particularmente com relação à ingestão energética aumentada no FDS em relação aos dias de semana. Haines et al. ${ }^{10}$, identificaram consumo superior de energia no FDS (de sexta-feira a domingo) para a amostra total avaliada, com aumento mais expressivo na faixa etária de 19 a 50 anos. Ademais, encontraram aumento no consumo de gordura e álcool para os adultos e diminuição da proporção de carboidrato ingerido no FDS em comparação aos dias de semana. Haines et al. ${ }^{10} \mathrm{e} \mathrm{Jula} \mathrm{et} \mathrm{al.}{ }^{14}$ consideraram como FDS o período compreendido entre sexta-feira e domingo. No presente estudo, foi utilizado o período de sábado a domingo, uma vez que o consumo de energia e macronutrientes referente à sexta-feira da população estudada apresentou-se semelhante ao consumo de segunda a quinta-feira.

O aumento na ingestão calórica da população brasileira nos dias de FDS foi em média de $153 \mathrm{kcal}$, o que pode levar a um incremento médio de $1.224 \mathrm{kcal}$ ao longo de um mês e quase $15.000 \mathrm{kcal}$ ao final de um ano. Esse acréscimo, se não for compensado com aumento do dispêndio de energia, pode levar ao ganho de $2 \mathrm{~kg}$ em um ano. Assim, essa variação na ingestão 
de energia segundo os dias de semana podem ser um fator que contribui para a elevação das prevalências de excesso de peso e obesidade registradas no país ${ }^{12,19}$. Outros estudos relacionaram o aumento na ingestão calórica nos finais de semana com a elevação do peso corporal $^{7,22}$. De Craemer et al. ${ }^{7}$, com objetivo de avaliar se alterações na dieta ou na prática de atividade física durante a semana contribuía para o ganho de peso ou para dificultar a perda de peso, desenvolveu uma revisão sistemática com pré-escolares e observaram ganho de peso nos dias de fim de semana, mas não nos dias de semana. Esse aumento de peso foi atribuído à maior ingestão energética e à redução da prática de atividade física no final de semana em relação aos dias da semana ${ }^{7}$.

Para Castro $^{5}$, as diferenças observadas no consumo alimentar entre dias de semana e de FDS podem ser atribuídas a mudanças na rotina diária. Durante a semana, o tempo destinado às refeições é, em geral, reduzido devido às obrigações de trabalho, estudo e outras tarefas ${ }^{5}$. No FDS, é possível que haja maior consumo fora de casa, maior frequência de comemorações e festas e maior tempo disponível para as refeições, condições que podem contribuir para o aumento do consumo no FDS 5,6,8.

Os resultados deste estudo são consistentes com as análises que avaliaram o consumo alimentar e a disponibilidade de alimentos no país. Pereira et al..$^{21}$ avaliaram dados desse mesmo inquérito e identificaram elevada participação de alimentos com teor excessivo de gorduras sólidas e açúcar de adição na dieta dos brasileiros. Levy et al. ${ }^{16}$, ao avaliarem a disponibilidade domiciliar de alimentos entre 1987 e 2003, encontraram que a disponibilidade domiciliar de açúcar de adição no Brasil atingiu 16,7\% do total de calorias disponíveis nos domicílios e essa participação mostrou-se elevada em todos os estratos regionais e de renda.

Adicionalmente, os resultados apresentados também são compatíveis com estudos que avaliaram o consumo alimentar por classes de renda, os quais têm observado que as classes de renda mais elevadas apresentam consumo mais expressivo de alimentos ricos em energia, como refrigerantes ${ }^{18,27}$. Nesta análise, os indivíduos de renda mais elevada $(\geq 5$ salários mínimos per capita) apresentaram aumento expressivo no consumo de bebidas com adição de açúcar no FDS, o que não ocorreu entre indivíduos do nível mais baixo de renda $(<0,5$ salários mínimo per capita), embora nesses tenha havido aumento da contribuição das gorduras para a ingestão diária de energia.

A análise baseada em um único dia de registro alimentar pode ser considerada uma limitação deste estudo. Entretanto, dados de um único dia de registro alimentar obtidos em amostra representativa da população são capazes de fornecer estimativas confiáveis de médias populacionais ${ }^{9}$. Ademais, a qualidade da informação no primeiro dia tem sido considerada superior àquela dos dias subsequentes de registros alimentares ${ }^{28}$. Optou-se por utilizar apenas o primeiro dia de registro alimentar porque, ao analisar alimentos, a proporção de zeros na resposta é muito elevada, o que impede um ajuste estatístico adequado ${ }^{15,26}$. Além disso, como a variável dietética é dependente no modelo, o efeito do erro aleatório (variabilidade intrapessoal) é de aumento da variância total, mas não influencia as diferenças entre as médias. $\mathrm{O}$ aumento da variância poderia levar à redução do poder da amostra ${ }^{3}$; porém, o tamanho amostral avaliado permite detectar diferenças nas proporções de consumo de grupos de alimentos e nas médias de ingestão de energia, nutrientes e grupos de alimentos com elevado poder e nível de confiança.

Como ponto forte deste estudo, destaca-se a representatividade da amostra em nível nacional. Outro aspecto que pode ser considerado um ponto forte deste estudo é a validade do método aplicado para a obtenção de dados de consumo alimentar, avaliado em estudo que adotou, como referência para comparação, a estimativa do gasto energético pela água duplamente marcada, o qual indicou que o sub-relato na ingestão de energia foi, aproximadamente, $30 \%{ }^{17}$.

Este estudo constatou que, no Brasil, ocorre aumento da ingestão de energia e de marcadores de uma alimentação relacionada a efeitos deletérios sobre a saúde durante os finais de semana, especialmente as bebidas com adição de açúcar. Paralelamente, nos finais de semana, ocorre redução do consumo de marcadores da alimentação saudável, como a combinação de arroz 
e feijão, hortaliças, e frutas. Esses achados devem ser alvo de estudos que considerem a repercussão dessas mudanças ao longo do tempo, uma vez que o aumento sistemático da ingestão energética pode levar ao desequilíbrio no balanço energético e favorecer o ganho de peso excessivo. Também, o incremento regular do consumo de açúcar de adição contido em bebidas pode aumentar o risco de desenvolvimento de distúrbios metabólicos, como a resistência à insulina e o diabetes. Essas evidências observadas no presente estudo devem ser consideradas na formulação de iniciativas de promoção da alimentação saudável, devendo ser ressaltada a importância da manutenção dos hábitos alimentares e de atividade física saudáveis também nos dias de final de semana.

\section{REFERÊNCIAS}

1. Bauer KW, Larson NI, Nelson MC, Story M, Neumark-Sztainer D. Fast food intake among adolescents: secular and longitudinal trends from 1999 to 2004. Prev Med. 2009;48(3):284-7. https://doi.org/10.1016/j.ypmed.2008.12.021

2. Beaton GH, Milner J, McGuire V, Feather TE, Little JA. Source of variance in 24-hour dietary recall data: implications for nutrition study design and interpretation: carbohydrate sources, vitamins, and minerals. Am J Clin Nutr. 1983;37(6):986-95.

3. Beaton $\mathrm{GH}$. Approaches to analysis of dietary data: relationship between planned analyses and choice of methodology. Am J Clin Nutr. 1994;59(1Suppl):253S-61S.

4. Brown WJ, Williams L, Ford JH, Ball K, Dobson AJ. Identifying the energy gap: magnitude and determinants of 5-year weight gain in midage women. Obes Res. 2005;13(8):143-41. https://doi.org/10.1038/oby.2005.173

5. Castro JM. Weekly rhythms of spontaneous nutrient intake and meal pattern of humans. Physiol Behav. 1991;50(4):729-38. https://doi.org/10.1016/0031-9384(91)90010-L

6. Collaço JHL. Um olhar antropológico sobre o hábito de comer fora. CAMPOS Rev Antropol Soc. 2003;4:171-94. https://doi.org/10.5380/cam.v4i0.1615

7. De Craemer M, De Decker E, De Bourdeaudhuij I, Vereecken C, Deforche B, Manios Y, et al. Correlates of energy balance-related behaviours in preschool children: a systematic review. Obes Rev. 2012;13 Suppl 1:13-28. https://doi.org 10.1111/j.1467-789X.2011.00941.x

8. Diez Garcia RW. Práticas e comportamento alimentar no meio urbano: um estudo no centro da cidade de São Paulo. Cad Saude Publica.1997;13(3):455-67. https://doi.org/10.1590/S0102311X1997000300021

9. Dodd KW, Guenther PM, Freedman LS, Subar AF, Kipnis V, Midthune D, et al. Statistical methods for estimating usual intake of nutrients and foods: a review of the theory. I Am Diet Assoc. 2006;106(10):1640-50. https://doi.org/10.1016/j.jada.2006.07.011

10. Haines PS, Hama MY, Guilkey DK, Popkin BM. Weekend eating in the United States Is linked with greater energy, fat, and alcohol Intake. Obes Res. 2003;11(8):945-9. https://doi.org/10.1038/oby.2003.130

11. Instituto Brasileiro de Geografia e Estatística. Pesquisa de Orçamentos Familiares 2008-2009: análise do consumo alimentar pessoal no Brasil. Rio de Janeiro: IBGE; 2011 [citado 4 abr 2017]. Disponível em: http://www.ibge.gov.br/home/estatistica/populacao/condicaodevida/ pof/2008_2009_analise_consumo/

12. Instituto Brasileiro de Geografia e Estatística. Pesquisa de Orçamentos Familiares 2008-2010: antropometria e estado nutricional de crianças, adolescentes e adultos no Brasil. Rio de Janeiro: IBGE; 2010. Disponível em: http://biblioteca.ibge.gov.br/visualizacao/livros/liv45419.pdf

13. Instituto Brasileiro de Geografia e Estatística. Pesquisa de Orçamentos Familiares 2008-2009: tabelas de composição nutricional dos alimentos consumidos no Brasil. Rio de Janeiro: IBGE; 2011 [citado 4 abr 2017]. Disponível em: http://biblioteca.ibge.gov.br/visualizacao/livros/liv50002.pdf

14. Jula A, Seppänen R, Alanen E. Influence of days of the week on reported food, macronutrient and alcohol intake among an adult population in south western Finland. Eur J Clin Nutr. 1999;53(10):808-12. https://doi.org/10.1038/sj.ejcn.1600853

15. Kipnis V, Midthune D, Buckman DW, Dodd KW, Guenther PM, Krebs-Smith SM, et al. Modeling data with excess zeros and measurement error: application to evaluating relationships between episodically consumed foods and health outcomes. Biometrics. 2009;65(4):1003-10. https://doi.org/10.1111/j.1541-0420.2009.01223.x 
16. Levy RB, Claro RM, Bandoni DH, Mondini L, Monteiro CA. Disponibilidade de "açúcares de adição" no Brasil: distribuição, fontes alimentares e tendência temporal. Rev Bras Epidemiol. 2012;15(1):3-12. https://doi.org/10.1590/S1415-790X2012000100001

17. Lopes TS, Luiz RR, Hoffman DJ, Ferriolli E, Pfrimer K, Moura AS, et al. Misreport of energy intake assessed with food records and 24-h recalls compared with total energy expenditure estimated with DLW. Eur J Clin Nutr. 2016;70(11):1259-64. https://doi.org/10.1038/ejcn.2016.85

18. Martins APB, Levy RB, Claro RM, Moubarac JC, Monteiro CA. Participação crescente de produtos ultraprocessados na dieta brasileira (1987-2009). Rev Saude Publica. 2013;47(4):656-65. https://doi.org/10.1590/S0034-8910.2013047004968

19. Ministério da Saúde (BR), Secretaria de Vigilância em Saúde, Departamento de Vigilância de Doenças e Agravos Não Transmissíveis e Promoção da Saúde. Vigitel Brasil 2014: vigilância de fatores de risco e proteção para doenças crônicas por Inquérito telefônico. Brasília (DF); 2015 [citado 4 abr 2017]. Disponível em: http://bvsms.saude.gov.br/bvs/publicacoes/vigitel_brasil_2014.pdfr

20. Moura EC, Silva SA, Malta DC, Morais Neto OL . Fatores de risco e proteção para doenças crônicas: vigilância por meio de inquérito telefônico, VIGITEL, Brasil, 2007. Cad Saude Publica. 2011;27(3):486-96. https://doi.org/10.1590/S0102-311X2011000300009

21. Pereira RA, Duffey KJ, Sichieri R, Popkin BM. Sources of excessive saturated fat, trans fat and sugar consumption in Brazil: an analysis of the first Brazilian nationwide individual dietary survey. Public Health Nutr. 2014;17(1):113-21. https://doi.org/10.1017/S1368980012004892

22. Racette SB, Weiss EP, Schechtman KB, Steger-May K, Villareal ET, Obert KA, et al. Influence of weekend lifestyle patterns on body weight. Obesity (Silver Spring). 2008;16(8):1826-30. https://doi.org/10.1038/oby.2008.320

23. Rothausen BW, Matthiessen J, Hoppe C, Brockhoff PB, Andersen LF, Tetens I. Differences in Danish children's diet quality on weekdays v. weekend days. Public Health Nutr. 2012;15(9):1653-60. https://doi.org/10.1017/S1368980012002674

24. Rothausen BW, Matthiessen J, Andersen LF, Brockhoff PB, Tetens I. Dietary patterns on weekdays and weekend days in 4-14-year-old Danish children. Br J Nutr. 2013;109(9):1704-13. https://doi.org/10.1017/S0007114512003662

25. Schmidt MI, Duncan BB, Silva GA, Menezes AM, Monteiro CA, Barreto SM, et al. Doenças crônicas não transmissíveis no Brasil: carga e desafios atuais. London: The Lancet; 2011 [citado 4 abr 2017]. (Saúde no Brasil, 4). Disponível em: http://actbr.org.br/uploads/conteudo/926_Doencas.pdf

26. Souverein OW, Dekkers AL, Geelen A, Haubrock J, Vries JH, Ocké MC, et al. Comparing four methods to estimate usual intake distributions. Eur J Clin Nutr. 2011;65 Suppl 1:S92-101. https://doi.org/10.1038/ejcn.2011.93

27. Souza AM, Pereiral RA, Yokoo EM, Levy RB, Sichieri R. Alimentos mais consumidos no Brasil: Inquérito Nacional de Alimentação 2008-2009. Rev Saude Publica. 2013;47 Supl 1:190S-9S. https://doi.org/10.1590/S003489102013000700005

28. Subar AF, Kipnis V, Troiano RP, Midthune D, Schoeller DA, Bingham S, et al. Using intake biomarkers to evaluate the extent of dietary misreporting in a large sample of adults: the OPEN Study. Am J Epidemiol. 2003;158(1):1-13. https://doi.org/10.1093/aje/kwg092

29. Willet W. Nutritional epidemiology. 3.ed. New York: Oxford University Press; 2013. (Monographs in Epidemiology and Biostatistics, 40).

Financiamento: Conselho Nacional de Desenvolvimento Científico e Tecnológico (CNPq - Processo 480296/2007-3).

Contribuição dos Autores: Analise e interpretação os dados: LSM, BKH, CCPE, AMS, EVJ, RS, RAP. Redação do manuscrito: LSM, BKH, CCPE, AMS, EVJ. Revisão do manuscrito. LSM, RS, RAP. Aprovar a versão final a ser publicada; RAP, RS. Assumir responsabilidade pública pelo conteúdo do artigo: LSM, RAP.

Conflito de Interesses: Os autores declaram não haver conflito de interesses. 